\title{
Information is a Physical Entity
}

\author{
Rolf Landauer \\ IBM Thomas J. Watson. Research Center \\ P. O. Box 218, Yorktoun Heights, NY 10598, U.S.A.
}

\begin{abstract}
This paper, associated with a broader conference talk on the fundamental physical limits of information handling, cmphasizes the aspects still least appreciated. Information is not an abstract entity but exists only through a physical representation, thus tying it to all the restrictions and possibilities of our real physical universe. The mathematician's vision of an unlimited sequence of totally reliable operations is unlikely to be implementable in this real universe. Speculative remarks about the possible impact of that, on the ultimate nature of the laws of physics are included.
\end{abstract}

\section{Introduction}

The fundamental physical limits of the computational process have been an object of study for some decades. A roadmap to the earlier work in this ficld was presented in [1]. At that time it had become clear that, contrary to widespread earlier discussions, the classical communications channel does not require a minimum energy expenditure per shipped bit. It had also been widely assumed that a quantum mechanical channel, designed to transmit classical bits (guaranteed to be in a 0 or 1 state rather than in a coherent superposition of 0 and 1) required a minimal energy per transmitted bit, proportional to the bit rate. Since the appearance of Ref. [1] it has been shown that such a quantum channel, whether transmitting classical bits or qubits (quantum superpositions of 0 and 1 ) does not require such a minimal energy expenditure per bit $[2,3]$.

The results presented by this author, concerning the energy requirements of the communications channel have, despite their conflict with earlier notions, attracted relatively little attention in the published literature. That arises, in part, from the recent emphasis on quantum computation and quantum parallelism [4]. That body of work has displaced questions concerning energy requirements. While the early proposals [5] for quantum computers viewed 
unclocked processes, activated by the system's initial kinetic energy, the more detailed recent proposals invoke continual external pulses, presumably requiring continual energy expenditure. Therefore, the proponents of quantum parallelism have deemphasized the earlier emphasis on minimal energy consumption. Rather than repeat, here, older discussions of reversible information handling, we emphasize in this brief written version, associated with a broader talk, those aspects of the subject which are still least understood and appreciated.

Information is inevitably inscribed in a physical medium. It is not an abstract entity. It can be denoted by a hole in a punched card, by the orientation of a nuclear spin, or by the pulses transmitted by a neuron. The quaint notion that information has an existence independent of its physical manifestation is still seriously advocated [6]. This concept, very likely, has its roots in the fact that we were aware of mental information long before we realized that it, too, utilized real physical degrees of freedom.

Our intuition has misled us in other ways. When we learned to count on our sticky and classical fingers, we naturally thought of information as classical. Today we understand that we can utilize a coherent quantum superposition of 0 and 1. This author has expressed skepticism $[3,7]$ about the practicality of quantum mechanical computation, in which data has to be subject to a great many successive steps. That differentiates quantum computation, with its implicit parallelism capability, from a communications link; in the latter each bit is likely to receive limited handling. Despite my reservations about practical utility, I welcome the quantum computation proposals and effort. They help to underline the central inessage addressed here: The physical nature of information ties it to all the restrictions and possibilities of our actual physical universe. This is a message passed by this author for many decades [8].

Our prevailing pattern of thought differs substantially from the point of view advocated here. There is a tendency to think of mathematics as a tool which somehow existed before and outside of our physical world. Mathematics, in turn, allowed the formulation of physical laws which then run the world, much as a process control computer runs a chemical plant. Here, instead, we emphasize that information handling has to be done in the real physical world, and the laws of physics exist as instructions for information handling in that real world. It, therefore, makes no sense to invoke operations, in the laws of physics, which are not executable, at least in principle, in our real physical world.

Our accepted laws of physics invoke continuum mathematics, which is, in turn, based on the notion that any required degree of precision can be obtained by invoking enough successive operations. But our real universe is unlikely 
to allow an unlimited sequence of totally reliable operations. The memory size is likely to be limited, perhaps, because the universe is limited. Even in an unlimited universe it is a strong presumption to invoke the possibility of assembling an arbitrarily large organized memory structure. Furthermore, in a world full of deleterious processes including noise, corrosion, electromigration, incident alpha particles and cosmic rays, earthquakes and spilled cups of coffee, it would be unreasonable to assume that each step in an unlimited sequence of operations can be carried out infallibly. Redundancy can be invoked to reduce error probabilities, but only to reduce, not to eliminate. Furthermore, error protection comes at the expense of extra apparatus and extra steps, thus using up the available parts of the universe more quickly.

What can replace the real number system and allow for the limitated precision of real physical operations? I do not know. The replacement does not necessarily lead to algorithms with a limited number of bits. The limited precision may appear in a more statistical fashion. The laws of physics are algorithms for calculation, and in their proper form must respect the limited information handling capabilities of the real universe. The reader may ask: "Does our limited ability to describe the physical world prevent that world from doing its own thing more precisely? Isn't it just our knowledge that is limited?" The answer: Behavior which cannot be followed, described, or observed, is not a matter of science. If I am told that seven angels are on the head of a pin and that angels are not detectable, I cannot call that an erroneous assertion. But it is not a matter for science.

The question arises: Can I not know that $2+2=4$ exactly, and not just to a very high degree of accuracy? If we want to prove $2+2=4$ within a closed postulate system describing the integers, or the real numbers, that requires a very limited number of successive steps, with each step depending on relatively few bits. The difficulties we have stressed will be minimal, and we can go through the required procedure with high probability that it was free of error. But the use of mathematics in physics requires us to go beyond operations within a closed postulate system. We need to do number crunching, and in that task have to invoke real physical information bearing degrees of freedom. That is where the problems that we have identified will turn up. We cannot expect to distinguish $\pi$, as defined by a series expansion, from some terribly close neighbor.

If we have only limited precision available, how does that bear on the laws of physics? One possibility relates to the ultimate source of irreversibility and of fluctuations in the real world, a world where we can readily observe departure from Hamiltonian behavior [9]. The lack of precision in the laws of physics is, essentially, a noise source. Furthermore, irreversibility is often explained by some sort of coarse-graining process. That is also supplied, directly and unavoidably, by the lack of precision. Limited precision may, furthermore, be 
the cause of the apparent classical behavior so conspicuously displayed around us. There are many proposed explanations for that, and we can cite only a few [10], including some counter-arguments. Some of these explanations may be correct, and there may not really be a need to say more. Nevertheless, a totally quantum mechanical behavior, in systems with some complexity, and followed for some time, requires a precise evaluation of phases for the competing histories, for the competing Feynman paths. In a world with limited precision relative phases will, eventually, get lost and this will lead to classical behavior. As in my discussion of noise and irreversibility, the limited precision acts as if the universe had an unpredictable environment with which it interacts.

Even if the very speculative ideas above related to irreversibility, noise, and apparently classical behavior, are wrong, there is a more basic underlying theme. Information handling takes place in the physical world and mathematics depends on that physical world, its laws and its parts. At a minimum, we caution those who invoke the wave function of the whole universe. Where is the second universe which is used to denote that state?

Our view that mathematics is part of the physical world can be used to resolve a longstanding supposed puzzle. There are many references to Wigner's remarks about "the unreasonable success of mathematics," e.g. Ref. [11]. That success, in characterizing the physical world is indeed a puzzle if we start from the presumption that mathematics existed before and exists apart from the physical universe. Instead, mathematics uses the physical universe, and is a part of physics. The utility of mathematics is related to the theme [11] that the laws of physics have a unity and are not separate at every possible level. Counting physical objects, pebbles, bones, steps, or people, was the start of mathematics. Kronecker got it wrong when he said that God made the integers, man made the rest. The integers were the beginning of our experimental observation of the universe.

My emphasis on the executability of operations on information is an enlargement of a view advocated by P. W. Bridgman, Nobel laureate, high pressure physicist, and author of a number of discussions of "operationalism." The latter can be considered to be a pragmatic Yankee variant of logical positivism. Logical positivism has largely been cast aside today. But Bridgman's humbler plea that science deal with specifiable and executable steps is surely acceptable common sense, even if we admit that a totally unambiguous specification is unlikely. Bridgman, in 1934, published a paper A Physicist's Second Reaction to Mengenlehre [12]. The journal that published the paper was oriented to fundamental questions in mathematics. The paper starts with a remarkable qualifying note by the editor: "The Scripta Mathematica takes pleasure in publishing this interesting article by Professor P. W. Bridgman. As in the case of all articles, however, it is understood that this does not necessarily 
represent the views of the editors." Bridgman was, by that time, already well known, and science is normally full of divergent viewpoints. Decades later, we can only guess that the unusual editorial qualification represented the editor's desperation. He may have been hesitant to turn down a famous scientist and, at the same time, was afraid of the wrath of his readers. Bridgman's paper presents his attempt to wrestle with the paradoxes of set theory. He objects to self-referential definitions and asks for mathematical procedures in which each successive step can be carried out directly and unambiguously. Bridgman rules out operations which depend upon information available only later on, i.e. depend on the outcome of the step being taken. I quote one of his illustrations:

Another example of the sort of situation which may arise when our terms do not have the proper amount of staticness is afforded by the example of the map of London, made on one of the flag stones of London itself. The difficulty is that the map itself, being a part of London, must be represented in the map, and this map of the map must again be represented, and so in unterminating progression. The point here is to be sought in the operational meaning of London. "London" is not a fixed and static thing, but itself changes as the action in question progresses, for the London with a map is not the same as the original London without a map. Here, therefore, by employing a non-static, fluent, object instead of the fixed object of Aristotelian convention, we find ourselves involved in an infinite process, which of course cannot be carried out actually, so that the corresponding physical object has no "existence."

Bridgman (in contrast to this author) is tolerant of an unlimited sequence of steps. He does not, however, allow for something else to be done after an infinite sequence. That rules out the famous diagonal procedure which appears in much of mathematics from Cantor to Gödel. No wonder the editor felt the need to insert a disclaimer. Incidentally, in the above quotation from Bridgman we can substitute "wave function of the universe" for "map of London."

In a remarkable coincidence(?) Bridgman uses the word program for such a succession of unambiguously executable instruction. He would have been entirely satisfied by a restriction of mathematics to that which can be done on a Turing machine, which came along a few years later. Indeed Bridgman's paper can be viewed as a want ad for a Turing machine. Bridgman did not go on to ask about the physical realizability of the operations in such a machine. That extension is the point of Ref. 8 and of this discussion. Bridgman's notions, of course, are not totally unrelated to reservations put forth by others interested in the foundations of mathematics [13], e.g. intuitionism, but do not seem to match any of those all that closely. 


\section{References}

[1] R. Landauer, in: Proceedings Workshop on Physics and Computation, PhysComp'94, IEEE Comp. Soc. Press, Los Alamitos, 1994, p. 54.

[2] R. Landauer, Science 272 (1996) 188.

[3] R. Landauer, Proc. R. Soc. Lond. A 454 (1998) 305.

[4] D. DiVincenzo, E. Knill, R. Laflamme, and W. Zurek (Eds.), Quantum Coherence and Decoherence, Proc. R. Soc. Lond. A 454 (1998) 257-486.

[5] P. Benioff, J. Stat. Phys. 22 (1980) 563; P. Benioff, J. Stat. Phys. 29 (1982) 515; P. Benioff, Phys. Rev. Lett. 48 (1982) 1581; R. P. Feynman, Optics News 11 (1985) 11; reprinted in Found. Phys. 16 (1986) 507.

[6] R. Penrose, The Emperor's New Mind, Oxford University Press, Oxford, 1989.

[7] R. Landauer, IEEE Transactions on Electronic Devices 43 (1996) 1637.

[8] R. Landauer, IEEE Spectrum 4 (1967) 105; reprinted in Speculations in Science and Technology 10 (1987) 292.

[9] R. Landauer, in: S. Haykin (Ed.), Selected Topics in Signal Processing, Prentice-Hall, Englewood Cliffs, 1989, p. 18; R. Landauer, Phys. B 68 (1987) 217.

[10] R. B. Griffiths, J. Stat. Phys. 36 (1984) 219; M. Gell-Mann and J. B. Hartle, in: Complexity, Entropy, and the Physics of Information, W. Zurek (Ed.), Addison-Wesley, Reading, 1990, p. 425; R. Omnès, The Interpretation of Quantum Mechanics, Princeton University Press, Princeton, 1994; R. Omnès, Rev. Mod. Phys. 64 (1992) 339; M. Gell-Mann and J. B. Hartle, in: Proceedings of the 3rd International Symposium on the Foundations of Quantum Mechanics in the Light of New Technology, S. Kobayashi, H. Ezawa, Y. Murayama, and S. Nomura (Eds.), Physical Society of Japan, Tokyo, 1990; N. Yamada, Phys. Rev. A 54 (1996) 182; W. H. Zurek and J. P. Paz, Il Nuovo Cimento 110B (1995) 611; W. H. Zurek, in: Physical Origins of Time Asymmetry, J. J. Halliwell, J. Pérez-Mercader, and W. H. Zurek (Eds.), Cambridge University Press, Cambridge, 1994, p. 175; W. H. Zurek, Physics Today 46 (1993) 81; C. H. Woo, Merging Histories and the Second Law, unpublished; F. Dowker and A. Kent, J. Stat. Phys. 82 (1996) 1575.F.

[11] F. Wilczek, Physics Today 51 (January 1998) 11.

[12] P. W. Bridgman, Scripta Mathematica 2 (1934) 3.

[13] R. Hersh, What is Mathematics, Really?, Oxford University Press, New York, 1997. 